\title{
The ABCs of SMC proteins: two-armed ATPases for chromosome condensation, cohesion, and repair
}

\author{
Tatsuya Hirano \\ Cold Spring Harbor Laboratory, Cold Spring Harbor, New York 11724, USA
}

The first draft of the human genome sequence was reported a year ago. It may be a good time to remind ourselves that the genetic information encoded in the $\sim 3000-\mathrm{Mb}$ sequence is stored not only in the public or private databases but also in the tiny space of the cell nucleus. The total length of human genomic DNA, which resides in 23 chromosomes, reaches approximately one meter. It is by no means a simple task to fold up the long DNA molecules and package them within a cell nucleus whose diameter is only $\sim 10 \mu \mathrm{m}$. Even more striking is that the DNA molecules are faithfully duplicated and segregated into two daughter cells in an extremely limited space. Although more than 100 years have passed since Walther Flemming first described the dynamic behavior of chromosomes (or mitosis) during cell division, it remains highly mysterious how this remarkable process of chromosome segregation is achieved at a mechanistic level. From a cytological point of view, two dramatic events occur on chromosomes during mitosis. The first one is the conversion of an amorphous mass of interphase chromatin into a discrete set of rodshaped chromosomes (chromosome condensation), which occurs from prophase to metaphase (Koshland and Strunnikov 1996; Hirano 2000). The second is the splitting of chromosomes into two halves, which takes place highly synchronously at the onset of anaphase (Dej and Orr-Weaver 2000; Nasmyth et al. 2000). As a crucial prerequisite for these events, duplicated chromosomes (sister chromatids) must be held together immediately after DNA replication in $S$ phase and throughout $G_{2}$ phase. The importance of this process (sister chromatid cohesion) has been fully appreciated only recently because the pairing of sister chromatids cannot be visualized by classical cytology before chromosomes condense in early mitosis. Recent genetic and biochemical studies have begun to shed light on the molecular mechanisms underlying cohesion, condensation, and separation of chromosomes during the mitotic cell cycle. One of the unexpected findings is that chromosome condensation and sister chromatid cohesion are regulated by distinct, yet

E-MAIL hirano@cshl.org; FAX (516) 367-8815.

Article and publication are at http://www.genesdev.org/cgi/doi/10.1101/ gad.955102. structurally similar, protein complexes termed condensin and cohesin, respectively. At the heart of the two protein complexes lie members of a family of chromosomal ATPases, the structural maintenance of chromosomes (SMC) family. Equally intriguing, SMC proteins are found in most, if not all, bacterial and archaeal species, implicating that their fundamental contribution to chromosome dynamics started even before the acquisition of histones during evolution. The goal of this review article is to discuss the current understanding of higherorder chromosome dynamics with an emphasis on the role of SMC proteins. I start with the basic description and classification of SMC proteins and then summarize emerging information on the diverse chromosomal functions supported by SMC proteins. Finally, I discuss the mechanistic aspects of bacterial and eukaryotic SMC proteins and try to make an integrated picture of their seemingly different actions.

\section{Basic architecture and classification of SMC proteins}

The primary structure of SMC proteins, which is shared from bacteria to humans, consists of five distinct domains (Fig. 1A). Two nucleotide-binding motifs, the Walker A and Walker B motifs, are located in the highly conserved $\mathrm{N}$-terminal and $\mathrm{C}$-terminal domains, respectively. The central domain is composed of a moderately conserved "hinge" sequence that is flanked by two long coiled-coil motifs. SMC proteins form homodimers or heterodimers. An electron microscopy (EM) study of a bacterial SMC homodimer showed that the coiled-coil motifs are arranged in an antiparallel fashion to make a two-armed, symmetrical structure (Fig. 1B; Melby et al. 1998). The hydrodynamic properties of the SMC dimer are consistent with the idea that the central hinge is actually flexible and allows opening and closing of the two arms (Hirano et al. 2001). This antiparallel configuration predicts that the $\mathrm{N}$-terminal and $\mathrm{C}$-terminal domains associate with each other to assemble a globular structure at each end of an SMC dimer. A recent crystallographic study has confirmed the formation of this catalytic domain in which the Walker A and Walker B motifs make close contact (Lowe et al. 2001). Site-di- 
Figure 1. Basic architecture of SMC proteins. (A) Primary structure of SMC proteins. The SMC monomer is a large polypeptide (between 1000 and 1400 amino acids). The $\mathrm{N}$-terminal ( 160 amino acids) and C-terminal ( 150 amino acids) domains are highly conserved, and contain the nucleotide-binding Walker $\mathrm{A}$ and Walker B motifs, respectively. The central domain is composed of two long coiled-coil regions (between 300 and 350 amino acids) and a nonhelical hinge sequence $(\sim 200$ amino acids). (B) A rotary shadowing image of the Bacillus subtilis SMC homodimer (reproduced from I. Cell Biol., 1998, 142: 1595-1604, by copyright permission of The Rockefeller University Press). (C) Two models for dimerization of SMC proteins. Coiled-coil interactions betw een two different subunits may mediate dimerization (left). Alternatively, two self-folded subunits may dimerize by a hinge-mediated interaction (right). Note that, in both cases, the two arms are composed of antiparallel coiled coils.

rected mutagenesis has shown that both motifs contribute to ATP binding and hydrolysis (Hirano et al. 2001). Despite the progress in our understanding of the architecture of SMC proteins, it remains to be determined how two polypeptides are folded to make an SMC dimer. Two models have been proposed so far. First, dimerization may be mediated by coiled-coil interactions between the two different subunits (Fig. 1C, left; Melby et al. 1998). Alternatively, the two subunits may be selffolded to form two separate coiled-coil rods, which, in turn, dimerize by a hinge-mediated interaction (Fig. 1C, right; Hirano et al. 2001). It should be noted that conventional EM does not distinguish between the two models because they predict a virtually identical architecture of the coiled-coil arms. Further analysis is required to clarify this important issue.

Most of the bacterial and archaeal genomes contain a single smc gene. The minimal functional unit of the gene products is likely to be a homodimer, as has been shown for the SMC protein from the Gram-positive bacterium Bacillus subtilis (Fig. 2, left; Hirano and Hirano 1998; Melby et al. 1998). Although a subclass of
Gram-negative bacteria including Escherichia coli lack SMC proteins, a gene product called MukB plays an analogous cellular function to that of SMCs (for review, see Hiraga 2000). In eukaryotes, at least six members of the SMC protein family are found in individual organisms. Because each of them has a specific partner with which to form an SMC heterodimer, eukaryotic SMC heterodimers can be classified into three distinct groups: SMC1-SMC3, SMC2-SMC4, and SMC5-SMC6 (Table 1). These heterodimers further associate with different sets of non-SMC subunits to assemble fully functional SMC holocomplexes. Both SMC and non-SMC subunits appear to contribute to the acquisition of distinct biochemical and cellular functions of different SMC holocomplexes.

\section{SMC2-SMC4: compacting chromosomes}

Condensin and mitotic chromosome condensation

The holocomplex of condensin (also called 13S conden$\sin$ ) is composed of two SMC subunits (SMC2/CAP-E
BsSMC

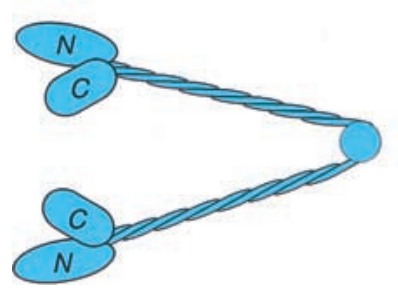

condensin

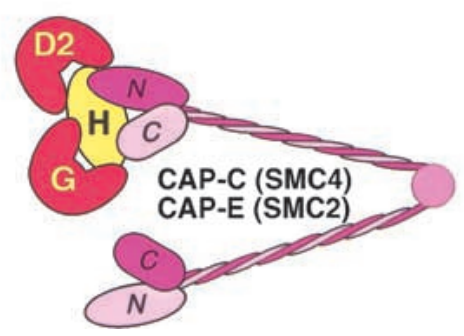

\section{cohesin}

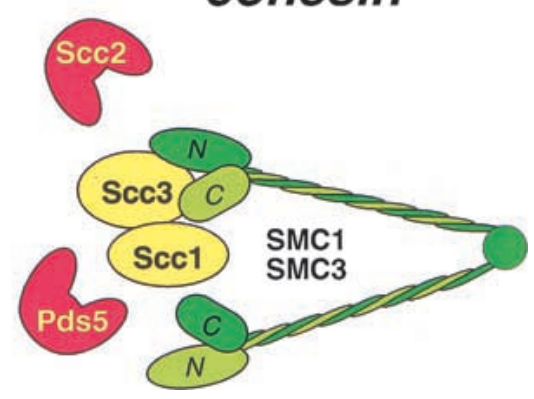

Figure 2. Subunit organization of a bacterial SMC protein (BsSMC), and eukaryotic condensin and cohesin complexes. SMC dimers are shown by the V-shaped, two-armed structures. The positions of the non-SMC subunits are arbitrary. Proteins with HEAT repeats are shown in red, and other non-SMC subunits are shown in yellow. The HEAT proteins Scc2 and Pds5 cooperate with cohesin to support sister chromatid cohesion although neither of them is a stoichiometric subunit of the cohesin complex. 
Table 1. Components of eukaryotic SMC protein complexes

\begin{tabular}{|c|c|c|c|c|c|c|c|}
\hline Subunits & S. cerevisiae & S. pombe & C. elegans & D. melanogaster & A. thaliana & $X$. laevis & H. sapiens \\
\hline \multicolumn{8}{|l|}{ Condensin } \\
\hline SMC2 & Smc2 & Cut14 & MIX-1 & DmSMC2 & BAB11491 & XCAP-E & hCAP-E \\
\hline SMC4 & Smc4 & Cut3 & F35G12.8 & DmSMC4/gluon & BAB10693 & XCAP-C & hCAP-C \\
\hline non-SMC & Ycs4 & Cndl & $?$ & CG1911 & CAB72176 & XCAP-D2/Eg7 & hCAP-D2/CNAP1 \\
\hline non-SMC & Ycs5/Ycg1 & Cnd3 & $?$ & CG17054 & ВАВ08309 & XCAP-G & hCAP-G \\
\hline non-SMC & Brnl & Cnd2 & $?$ & Barren & AAC25941 & XCAP-H & hCAP-H \\
\hline SMC4 variant & - & - & DPY-27 & - & - & - & - \\
\hline \multicolumn{8}{|l|}{ Cohesin } \\
\hline SMC1 & Smcl & Psm 1 & F28B34.7 & DmSMC1 & CAB77587 & XSMC1 & $\mathrm{hSMCl} 1 \alpha$ \\
\hline SMC3 & Smc3 & Psm3 & Y47D3A & DmSMC3/Cap & AAD26882 & XSMC3 & hSMC3 \\
\hline non-SMC & Scc1/Mcd1 & $\operatorname{Rad} 21$ & $\mathrm{COH}-1,2,3^{\mathrm{a}}$ & DmRAD21 & $>3$ homologs & XRAD21 & hRAD21 \\
\hline non-SMC & $\operatorname{Scc} 3$ & $\operatorname{Psc}^{\mathrm{b}}$ & $\mathrm{F} 18 \mathrm{E} 2.3$ & DmSA & CAB45374 & XSA1 and XSA2 & hSA1 and hSA2 \\
\hline SMC1 $\beta$ (meiotic) & - & - & - & - & - & ? & $\mathrm{hSMC} 1 \beta$ \\
\hline non-SMC (meiotic) & Rec8 & Rec8 & REC-8 & $?$ & SYN1/DIF1 & ? & Rec8 \\
\hline non-SMC (meiotic) & - & Rec11 & $?$ & CG13916? & $?$ & $?$ & STAG3/hSA3 \\
\hline \multicolumn{8}{|l|}{$\underline{\text { SMC5-6 complex }}^{\mathrm{c}}$} \\
\hline SMC5 & YOL034w & Spr18 & C27A2.1 & CG7783 & CAC01791(MSS2) & AW638169 (est) & hSMC5 \\
\hline SMC6 & Rhc18 & $\operatorname{Rad} 18$ & $\mathrm{C} 23 \mathrm{H} 4.6$ & CG5524 & MIM & BG160113 (est)? & hSMC6 \\
\hline
\end{tabular}

${ }^{\mathrm{a}} \mathrm{COH}-2$ and $\mathrm{COH}-3$ may have meiotic roles in the germ line (Pasierbek et al. 2001).

${ }^{b}$ Psc3 is not tightly associated with the other cohesion subunits in S. pombe (Tomonaga et al. 2000).

${ }^{\mathrm{c}}$ This complex contains other subunits whose identities remain to be determined (Fousteri and Lehman 2000).

and SMC4/CAP-C) and three non-SMC subunits (CAPD2, CAP-G, and CAP-H; Fig. 2, center). This five-subunit complex was originally identified in Xenopus laevis (Hirano et al. 1997) and subsequently found in different organisms including Schizosaccharomyces pombe (Sutani et al. 1999), Saccharomyces cerevisiae (Freeman et al. 2000), and Homo sapiens (Table 1; Schmiesing et al. 2000; Kimura et al. 2001). Two of the non-SMC subunits, CAP-D2 and CAP-G, share a structural motif called the HEAT repeats (Neuwald and Hirano 2000). The HEAT repeats are tandem repeats of an $\alpha$-helical structural unit that create a protein-recognition interface with an extended solenoidal shape (for review, see Kobe and Kajava 2000). They have been found in a number of proteins with diverse functions, including nuclear transport (importin $\beta$ ) and transcriptional control (TAF-172/ Mot1). Interestingly, Scc2/Mis4 and Pds5/BimD/Spo76, two gene products genetically implicated in sister chromatid cohesion, also contain HEAT repeats (Neuwald and Hirano 2000; Panizza et al. 2000). Although neither of them is a stoichiometric subunit of the cohesin complex, they cooperate with cohesin to establish and maintain sister chromatid cohesion, further emphasizing the structural (and possibly functional) similarity between the condensation and cohesion machineries (see below).

In Xenopus egg cell-free extracts, the condensin complex binds to chromosomes in a mitosis-specific manner, and is required for the establishment and maintenance of chromosome condensation (Hirano and Mitchison 1994; Hirano et al. 1997). Neither the SMC heterodimer nor the non-SMC subcomplex alone is able to induce chromosome condensation in the cell-free extracts, empha- sizing the functional importance of the five-subunit holocomplex (Kimura and Hirano 2000). Genetic studies of the condensin components have been reported from many organisms including $S$. cerevisiae (Strunnikov et al. 1995; Freeman et al. 2000; Lavoie et al. 2000; Ouspenski et al. 2000), S. pombe (Saka et al. 1994; Sutani et al. 1999), Caenorhabditis elegans (Lieb et al. 1998), and Drosophila melanogaster (Bhat et al. 1996; Steffensen et al. 2001). Each one of the five subunits is essential for cell viability in yeast, and this is most likely the case in all organisms. One of the most prominent phenotypes commonly observed in the condensin mutants is a severe defect in chromosome segregation during anaphase. The mass of chromosomes is pulled apart by the mitotic spindle, but they fail to segregate properly, exhibiting the so-called anaphase bridges. This phenotype is similar, if not identical, to that observed in mutants defective in topoisomerase II, consistent with the idea that one important function of condensin-mediated compaction is to facilitate the resolution of sister chromatids catalyzed by topoisomerase II (Koshland and Strunnikov 1996; Hirano 2000).

The exact mechanism by which the condensin complex contributes to chromosome condensation remains to be determined. In Xenopus egg extracts, a fluffy and unresolved mass of chromatin is produced in the absence of condensin (Hirano et al. 1997). This phenotype is clearly distinct from that observed in topoisomerase IIdepleted extracts (Hirano and Mitchison 1993), emphasizing the distinct mechanistic contributions of condensin and topoisomerase II to chromosome assembly in vitro. Abnormal chromosome condensation is also a common phenotype observed in vivo in many condensin 
mutants, but the extent of condensation defect varies between different mutants and different organisms. In Drosophila SMC4 mutants, for example, the shortening of the longitudinal axis of chromosomes is apparently normal, resulting in the formation of "dumpy" chromosomes with unresolved sister chromatids (Steffensen et al. 2001). It remains to be determined whether this phenotype is specific to the mutations in the SMC4 subunit or whether the residual level of SMC4 activity in the mutant is sufficient to support the axial shortening of chromosomes. In the future, systematic phenotypic analyses of different mutants should address the specific roles of individual subunits in vivo. Reconstitution of subcomplexes in vitro and their functional assessment in Xenopus egg extracts should provide complementary information.

\section{Condensin and global gene regulation}

In addition to their essential contribution to mitotic chromosome condensation and segregation, the condensin subunits play important functions at non-mitotic stages of the cell cycle. The best-characterized example for such functions is dosage compensation in C. elegans (for review, see Meyer 2000). The SMC2 ortholog MIX-1 associates with DPY-27 (a variant form of SMC4) to form a dosage compensation complex along with additional subunits including DPY-26 and DPY-28 (Lieb et al. 1998). This complex is specifically targeted to both $\mathrm{X}$ chromosomes of hermaphrodites to repress the level of transcription by half. MIX-1 is also a component of the condensin complex that participates in mitotic chromosome condensation and segregation. It is of great interest to determine whether the dosage compensation machinery accomplishes chromosome-wide gene repression by using the same mechanism that drives chromosome condensation in mitosis.

In Drosophila, the polycomb group (PcG) proteins act on specialized cis-elements (polycomb response elements, PRE) to maintain the transcriptionally repressed state of homeotic genes. A recent study using chromatin immunoprecipitation assays has revealed that topoisomerase II and the condensin subunit Barren/CAP-H colocalize on DNA sequences including the PREs in the bithorax complex (Lupo et al. 2001). Moreover, genetic experiments have shown that Barren is required for gene silencing mediated by one of the PREs, Fab-7. Thus, the condensin subunit and PcG proteins appear to cooperate to maintain the silenced state of gene expression, possibly by assembling condensed heterochromatin-like structures.

In $S$. cerevisiae, condensin concentrates in the rDNA region during mitosis. Interestingly, this binding to rDNA persists in interphase, implying that condensin may have a specialized function in organizing this highly repetitive locus with properties of heterochromatin (Freeman et al. 2000). An apparent enrichment of condensin subunits in the nucleolus has also been reported in human cells (Cabello et al. 2001).
Histone H3 phosphorylation and chromosome condensation

Several different mechanisms have been shown to regulate condensin functions in vitro and in vivo (Kimura et al. 1998; Collas et al. 1999; Sutani et al. 1999; Steen et al. 2000; Kimura et al. 2001). These include enzymatic activation of condensin in Xenopus and humans, and mitosis-specific nuclear transport in $S$. pombe. Importantly, both of the seemingly different levels of regulation involve direct phosphorylation of condensin subunits by the master mitotic kinase cdc2.

In this review, I focus on the potential role of histone H3 phosphorylation in condensin recruitment and chromosome condensation. The N-terminal tail of histone H3 is phosphorylated at serine 10, highly coincidently with the onset of mitotic chromosome condensation. In Tetrahymena thermophila, substitution of the serine residue with alanine (S10A) affects chromosome condensation and segregation (Wei et al. 1999). Recent evidence suggests that aurora B (also known as Ipl1 in S. cerevisiae and AIR-2 in C. elegans) is likely to be the major kinase that is responsible for this specific phosphorylation (Hsu et al. 2000; Speliotes et al. 2000). RNA interference (RNAi) experiments in Drosophila also support this conclusion (Adams et al. 2001b; Giet and Glover 2001). In Aspergillus nidulans, another kinase known as NimA acts as a histone H3 kinase (De Souza et al. 2000). Mutation or depletion of these $\mathrm{H} 3$ kinases causes defects in multiple events in mitosis including chromosome segregation and cytokinesis (Speliotes et al. 2000; Adams et al. 2001b; Giet and Glover 2001).

How does a loss of $\mathrm{H} 3$ phosphorylation affect chromosome segregation? A popular model is that the modification may send a signal to initiate chromosome condensation. The phosphorylated tail of histone $\mathrm{H} 3$ could function as a receptor that recruits chromosome condensation proteins such as the condensin complex (Wei et al. 1999). Consistent with this idea, a non-SMC subunit of condensin, Barren, is not properly targeted to chromosomes when aurora B is depleted by RNAi in Drosophila (Giet and Glover 2001). It is unclear, however, whether this is a direct consequence of the failure of $\mathrm{H} 3$ phosphorylation or an indirect effect of other problems caused by the absence of aurora B activity. In a purified system, for example, phosphorylation of histone $\mathrm{H} 3$ at serine 10 has little impact on the interaction between condensin and nucleosomes (Kimura and Hirano 2000). Moreover, in Xenopus egg extracts, condensin can interact with "tailless" nucleosomes (de la Barre et al. 2000), and artificial induction of $\mathrm{H} 3$ phosphorylation is not sufficient to recruit condensin to chromosomes (Murnion et al. 2001). Finally and most importantly, a recent study shows that neither chromosome condensation nor chromosomal targeting of condensin is compromised when H3 phosphorylation is drastically reduced by depletion of aurora B from the extracts (MacCallum et al. 2002). Thus, the exact role of this modification in chromosome dynamics remains elusive. In addition to serine 10 , serine 28 of histone $\mathrm{H} 3$ is phosphorylated in a mitosis-spe- 
cific manner (Goto et al. 1999). Unlike Tetrahymena, single or double mutations in these phosphorylation sites in S. cerevisiae cause no detectable defects in chromosome segregation (Hsu et al. 2000), providing an additional complexity to this problem. It is possible that combinatorial modifications of different histone tails are important for regulating chromosome behavior in mitosis, as is the case in transcriptional regulation (for review, see Stahl and Allis 2000).

A recent series of biochemical, cytological, and genetic studies strongly suggests that aurora B functions together with inner centromere protein (INCENP) in a protein complex (Adams et al. 2000, 2001a; Kaitna et al. $2000)$ that may also contain a small protein called survivin/BIR-1 (Speliotes et al. 2000; Uren et al. 2000; Morishita et al. 2001; Wheatley et al. 2001). These three proteins are collectively referred to as chromosomal passengers on the basis of their dynamic and characteristic localization during mitosis. The chromosomal passengers are associated with chromosome arms during the early stages of mitosis and accumulate progressively at inner centromeres by metaphase. They leave chromosomes in anaphase, redistributing to the spindle midzone and equatorial cortex. Both INCENP and survivin/BIR-1 are required for the proper localization of aurora B. Given this dynamic behavior, it is not surprising to find that the loss-of-function mutation of this class of proteins causes highly complex phenotypes. Conceivably, histone $\mathrm{H} 3$ is only one of the many substrates that are phosphorylated by aurora B during mitosis, and identification of nonhistone substrates is one of the important future directions. It is unknown whether condensin subunits are among the substrates of the aurora B-INCENP complex.

\section{The SUMO pathway and chromosome condensation: a potential link?}

SUMO (small ubbiquitin-related modifier) is a conserved ubiquitin-like small protein that is covalently attached to other proteins to modulate their functions (for review, see Melchior 2000). Recent studies point out a potential link between this posttranslational modification pathway and chromosome condensation. In S. cerevisiae, the temperature sensitivity of a condensin mutant, $s m c 2$, is suppressed by overexpression of Smt4, a protease that possesses SUMO-cleavage activity (Strunnikov et al. 2001). Smt4 is not an essential protein, but its null mutation decreases the fidelity of chromosome segregation and affects mitosis-specific targeting of condensin to rDNA. The slow-growth phenotype of $s m c 4 \Delta$ is suppressed by overexpression of Siz1, a protein that promotes SUMO conjugation in vitro (Johnson and Gupta 2001). In Drosophila, mutations in the Su(var)2-10 locus, which encodes a Sizl homolog, cause chromosome transmission defects and abnormal chromosome morphologies (Hari et al. 2001). On the other hand, a mutation of a component of the ubiquitin ligase CUL-2 causes defects in chromosome condensation in C. elegans (Feng et al. 1999). Thus, the currently available data are all intriguing but fragmentary. Future work should address how the SUMO (and ubiquitin) pathway might directly (or indirectly) affect the condensation machinery in these organisms.

\section{SMC1-SMC3: holding chromatids together}

\section{Cohesin and its interacting proteins}

The cohesin complex consists of the heterodimer of SMC1 and SMC3 and at least two non-SMC subunits (Scc1/Mcd1/RAD21 and Scc3/SAs; Fig. 2, right). The subunits of cohesin were systematically identified in $S$. cerevisiae by a genetic screen for mutants that display premature separation of sister chromatids (Michaelis et al. 1997). Some of them were identified independently by screens for mutants that affect proper segregation of mitotic chromosomes (Strunnikov et al. 1993; Guacci et al. 1997). The protein complex containing the corresponding gene products was found in Xenopus (Losada et al. 1998), and later in other organisms including S. cerevisiae (Toth et al. 1999), S. pombe (Tomonaga et al. 2000), and humans (Table 1; Losada et al. 2000; Sumara et al. 2000). In higher eukaryotic cells, several different isotypes are found in each of the two non-SMC subunits. For example, vertebrate cells have three Scc3/SA homologs. SA1 and SA2 form distinct complexes termed cohesin $^{\mathrm{SA} 1}$ and cohesin ${ }^{\mathrm{SA} 2}$ in mitotic cells (Losada et al. 2000; Sumara et al. 2000), whereas the third homolog, SA3/STAG3, has a meiosis-specific function (Pezzi et al. 2000; see below). The C. elegans genome has four Scc1 homologs whose functions are differentially regulated during development (Pasierbek et al. 2001).

Chromosomal binding sites of cohesin have been mapped in S. cerevisiae by chromatin immunoprecipitation assays. Cohesin associates with specific regions near centromeres and along chromosome arms with a preference for AT-rich sequences. In the arms, cohesin distributes with a periodicity of $\sim 15 \mathrm{~kb}$, as judged by a chromosome-wide hybridization approach (Blat and Kleckner 1999|, or of $\sim 9 \mathrm{~kb}$, as revealed by high-resolution chromosome walking (Laloraya et al. 2000). There is no apparent correlation between replication origins and the cohesin-binding sites. The association of cohesin with centromeres requires functional kinetochore proteins (Tanaka et al. 1999) and increases in mitotically arrested cells (Laloraya et al. 2000), suggesting that cohesin binding may be regulated differentially at centromeres and chromosome arms. In $S$. pombe, it has been shown recently that Swi6, a counterpart of the heterochromatin protein HP1, plays a role in recruiting cohesin specifically at centromeres (Bernard et al. 2001).

In both yeast and Xenopus, the loading of cohesin onto chromatin in $G_{1}$ is functionally separable from the establishment of sister chromatid cohesion in $S$ phase (Losada et al. 1998; Uhlmann and Nasmyth 1998). In S. cerevisiae, the HEAT protein Scc2 associates with Scc4 to form a complex required for the loading of cohesin onto chromatin (Ciosk et al. 2000). In S. pombe, Mis4 plays a role analogous to Scc2 (Tomonaga et al. 2000). 
How this loading process is achieved is still unknown. Another HEAT protein called Pds5 is required for the establishment and maintenance of cohesion in S. cerevisiae (Hartman et al. 2000; Panizza et al. 2000). The role of this class of proteins in other organisms (Pds5 in $S$. pombe, BimD in A. nidulans, and Spo76 in Sordaria macrospora) is far less clear, although several studies have shown that Pds5/BimD interacts genetically and physically with the cohesin complex (Holt and May 1996; Sumara et al. 2000; Tanaka et al. 2001). For example, Pds5 is not essential for mitotic growth in $S$. pombe under normal conditions (Tanaka et al. 2001), nor is BimD in $A$. nidulans at low temperatures (van Heemst et al. 2001). In Sordaria, mutations in Spo76 cause only subtle defects in the mitotic cell cycle, whereas they display prominent phenotypes in meiotic chromosome morphogenesis (van Heemst et al. 1999). Interestingly, when Pds5 is deleted, $S$. pombe cells become viable even in the absence of the cohesion protein Esol (see below) that is otherwise essential for mitotic growth (Tanaka et al. 2001). Further work will be required to determine the biochemical functions of these HEAT proteins and to clarify the seemingly diverse mutant phenotypes in different organisms.

\section{Functional coupling between DNA replication and sister chromatid cohesion}

Sister chromatid cohesion is established during S phase. Recent studies have begun to address the question of how cohesion factors functionally interact with the DNA replication machinery. In $S$. cerevisiae, Ctf7/Ecol is required for the establishment but not for the maintenance of cohesion, and genetically interacts with the sliding clamp PCNA (Pol30) and its putative loader Ctf18 (Skibbens et al. 1999; Toth et al. 1999). The binding of cohesin to chromatin is apparently normal in ctf7/ eco1 mutants. Intriguingly, Ctf7/Eco1-like sequences are present in highly variable forms among different organisms. For example, $S$. pombe Esol is composed of an Ecol-related domain essential for cohesion, and a DNA polymerase $\eta$-related domain implicated in translesion DNA synthesis (Tanaka et al. 2000). The Drosophila homolog displays a different chimeric organization. More recent studies in $S$. cerevisiae have provided additional evidence for the direct link between the DNA replication machinery and sister chromatid cohesion. First, a novel DNA polymerase activity (Pol $\sigma$, renamed from Pol к) is associated with Trf4, a protein involved in cohesion (Wang et al. 2000). Second, the establishment of proper cohesion requires an alternative clamp loader containing Ctf18, Ctf8, and Dcc1, as well as another protein, Ctf4, that physically interacts with the catalytic subunit of DNA polymerase $\alpha$ (Hanna et al. 2001; Mayer et al. 2001). On the basis of these results, a polymerase switching model has been proposed in which these replication factors may be dedicated specifically to replicate cohesin-associated regions of chromosomal DNA. Therefore, the replication-coupled establishment of cohesion appears far more complex than previously anticipated, even in a simple organism like $S$. cerevisiae. Fu- ture efforts should address the biochemical mechanism by which the passage of replication forks directs the construction of a physical bridge between newly synthesized DNA strands. It is tempting to speculate that this process accompanies a conformational change of the cohesin complex or its enzymatic activation.

\section{Unloading of cohesin and sister chromatid separation}

An elegant series of genetic and biochemical experiments in S. cerevisiae has shown that the cysteine protease Esp1 (or separase) cleaves the cohesin subunit Scc1, thereby promoting sister chromatid separation at the onset of anaphase (Uhlmann et al. 1999, 2000). Phosphorylation of Scc1 by the Polo/Cdc5 kinase enhances this cleavage reaction (Alexandru et al. 2001). A similar scheme is likely to operate in other eukaryotes as well. A small fraction of Scc1/RAD21 is cleaved during anaphase in S. pombe (Tomonaga et al. 2000) and human cells (Waizenegger et al. 2000). Moreover, ectopic expression of cleavage-resistant forms of Scc1/RAD21 disturbs chromosome segregation in these organisms (Tomonaga et al. 2000; Hauf et al. 2001), as has been shown in $S$. cerevisiae (Uhlmann et al. 1999).

Despite the conserved mechanism involving cohesin cleavage in anaphase, a striking difference exists in the regulation of sister chromatid cohesion between $S$. cerevisiae and higher eukaryotic cells. Unlike in yeast, most cohesin ( $95 \%$ ) dissociates from chromatin during prophase, far before the onset of anaphase, in metazoan cells (Losada et al. 1998, 2000; Sumara et al. 2000; Waizenegger et al. 2000). The exact mechanism of this prophase dissociation is unknown, although there is an indication that phosphorylation of the cohesin subunit SA/ Scc3 might be part of it (Losada et al. 2000). The remaining $\sim 5 \%$ of cohesin is apparently enriched in the centromere-proximal region under mitotically arrested conditions (Waizenegger et al. 2000; Warren et al. 2000; Hoque and Ishikawa 2001), leading to the proposal that cohesin dissociates first from chromosome arms during prophase and then from centromeres in anaphase. It remains to be determined, however, exactly how the temporal and spatial dissociation of cohesin is regulated in normal mitosis, in which chromosome arms are also held together until the onset of anaphase.

Why does cohesin dissociate from chromatin during prophase in metazoan cells? It is reasonable to speculate that this partial loss of cohesion is a prerequisite to the initiation of condensin-mediated condensation in prophase. Conceivably, a high density of cohesin on a chromosome arm limits the size of chromatin loops and thereby constrains the action of condensin in folding and compacting each loop. On the basis of this idea, it has been proposed that the shape of the metaphase chromosome is determined by a precise balance between the cohesion and condensation machineries (Losada and Hirano 2001b). In higher eukaryotes with large genomes, cohesion along chromosome arms must be released to allow efficient condensation, and loosening of arm cohe- 
sion is counterbalanced by an increased cohesion around centromeres.

\section{Meiosis-specific cohesin components}

Given the fundamental role of cohesin in sister chromatid cohesion during mitosis, it is not surprising to find that the cohesin subunits and other cohesion factors play vital roles in meiotic chromosome pairing and segregation. Emerging lines of evidence suggest that eukaryotes have evolved meiosis-specific cohesin components to modify the preexisting mitotic program. The best studied example of such components is Rec8, which has a similarity to the cohesin subunit Scc1/RAD21 (Michaelis et al. 1997) and is conserved from yeast to humans (Table 1; Parisi et al. 1999). In S. cerevisiae, Rec8 and Smc3 colocalize to chromosomal cores in prophase I and dissociate from chromosome arms in metaphase I, but remain bound to centromeres until metaphase II (Klein et al. 1999). Rec8 function is essential for cohesion, formation of axial elements (AEs), and recombination. The cleavage of Rec8 by separase in anaphase I is necessary for the release of arm cohesion and thereby for the disjunction of homologous chromosomes (Buonomo et al. 2000). In S. pombe, it has been shown that Rec8 is required to establish reductional chromosome segregation in meiosis I (Watanabe and Nurse 1999), and that this function is primed during the premeiotic $S$ phase at the inner centromeric region (Watanabe et al. 2001). Rec8 homologs involved in meiotic chromosome pairing and disjunction have also been characterized in Arabidopsis thaliana (Bai et al. 1999; Bhatt et al. 1999) and C. elegans (Pasierbek et al. 2001).

The S. pombe genome has two Scc3/SA-like sequences, and one of them, Rec11, is involved in meiotic cohesion and recombination (Table 1; Krawchuk et al. 1999). In mammalian cells, a similar protein, STAG3 (also known as SA3), has been implicated in sister chromatid arm cohesion during meiosis I (Prieto et al. 2001). In $S$. cerevisiae, there is no meiotic counterpart that belongs to this class of cohesion factors.

More recently, a meiosis-specific SMC protein has been reported in mammalian cells (Revenkova et al. 2001). This newest member of the SMC family is most closely related with SMC1 (therefore named SMC1 $\beta$ ) and is not found in the genome of yeast, Drosophila, C. elegans, or Arabidopsis. It associates with SMC3 but not with the canonical SMC1 (or SMC1 $1 \alpha$ ). SMC1 $\alpha$ is loosely associated, in a punctate pattern, with the AEs of the synaptonemal complexes (SCs) at the pachytene stage (Eijpe et al. 2000), whereas SMC1 $\beta$ is more tightly and uniformly distributed along the AEs. Importantly, although $\mathrm{SMC} 1 \alpha$ dissociates from the chromatin in late prophase I, SMC1 $\beta$ remains at the centromeres until metaphase II. This behavior predicts that SMC1 $\beta$, not SMC1 $\alpha$, is responsible for maintaining cohesion between sister centromeres in meiosis II.

Another recent study in mammalian meiotic cells has provided additional insight into meiotic chromosome structure. Although cohesin forms a chromosomal core along the AEs in pachytene, the integrity of this core structure is apparently intact even in the absence of the AEs (Pelttari et al. 2001). It should be noted that the reverse may not be the case: cohesin function is required for proper formation of the AEs at least in S. cerevisiae (Klein et al. 1999). Intriguingly, the cohesin core, without the AEs, can recruit recombination proteins and promote synapsis between homologous chromosomes (Pelttari et al. 2001). Thus, the new study leaves a number of fundamental questions on the structural and functional basis of meiotic chromosome pairing, recombination, and segregation.

\section{SMC5-SMC6: linking DNA repair and checkpoint responses}

Phylogenetic analyses reveal that eukaryotic cells have two additional members of the SMC family (e.g., Cobbe and Heck 2000). Recent biochemical studies in S. pombe and human cells have shown that these two proteins (now called SMC5 and SMC6) form a protein complex along with additional subunits whose identity remains to be established (Fousteri and Lehmann 2000; Taylor et al. 2001). SMC6 was originally identified as the gene product of rad18 in $S$. pombe, whose mutation causes hypersensitivity to both UV and $\gamma$ radiation (Lehmann et al. 1995). Unlike other Rad gene products, Rad18/SMC6 is essential for mitotic growth in $S$. pombe, and this is also the case with SMC5 (also known as Spr18; Fousteri and Lehmann 2000). Rad18/SMC6 is required to maintain a checkpoint arrest after DNA damage, and it genetically interacts with Brc1, a nonessential protein that shares BRCT domains with the breast cancer susceptibility gene product BRCA1 (Verkade et al. 1999). Moreover, rad18 is synthetically lethal with a mutant of topoisomerase II, but not with mutants of condensin or cohesin. The SMC5-SMC6 protein complex is therefore likely to play a role in higher-order chromosome organization, independently of condensation and cohesion, that is essential for genomic integrity and DNA damage responses. In mammals, SMC5 and SMC6 are highly expressed in the testis and associate with the X-Y chromosome pair in the late stage of meiotic prophase (Taylor et al. 2001), implicating their additional functions in meiosis.

In Arabidopsis, a mutant of an SMC6 homolog (called $\mathrm{mim}$ ) is hypersensitive to a variety of DNA-damaging agents and is defective in intrachromosomal homologous recombination in somatic cells (Mengiste et al. 1999|. Surprisingly, the homozygous mim plants develop normally, providing the first example of an eukaryotic SMC mutant that does not affect the viability of an organism. It has been proposed that MIM plays an active role in homologous recombination by increasing accessibility of chromosomal DNA to the recombination machinery.

\section{Primordial SMCs: illuminating the evolution of chromosome dynamics}

A requirement for SMC proteins in bacterial chromosome partitioning has been shown in B. subtilis and 
Caulobacter crescentus. B. subtilis smc null mutants are temperature-sensitive in rich growth medium and show multiple phenotypes at permissive conditions, including abnormal nucleoid morphology, mislocalization of the origin region, and accumulation of anucleate cells (Britton et al. 1998; Graumann et al. 1998; Moriya et al. 1998; Britton and Grossman 1999; Graumann 2000). Similar phenotypes are observed in the null mutant of the smc gene in C. crescentus (Jensen and Shapiro 1999). Few anucleate cells are produced under permissive conditions in this species, however, implying that a cell cycle checkpoint operates to arrest mutant cells at a predivisional stage. Increasing lines of recent evidence suggest that, in E. coli, MukB acts as the functional homolog of SMC. Although the primary sequences of MukB and SMC show a very limited homology, the two proteins do share a remarkably similar two-armed structure, as judged by electron microscopy (Melby et al. 1998). Mutant phenotypes of $E$. coli $m u k B$ are almost indistinguishable from those observed in $B$. subtilis $s m c$ mutants (Niki et al. 1991). Unlike the B. subtilis SMC (BsSMC) dimer, the MukB dimer associates with two other proteins, MukE and MukF, to form a three-subunit protein complex (Yamazoe et al. 1999). Moreover, mukE and mukF mutants display similar phenotypes to that of $m u k B$, suggesting that the three gene products act in concert in vivo. Although no apparent homolog of MukE or MukF is found in the genome of $B$. subtilis, it will be important to know whether BsSMC functions together with loosely associated non-SMC subunits whose structures may be highly divergent from MukE and MukF.

A large number of proteins have been shown to interact genetically with MukB (for review, see Hiraga 2000). A recent important finding is that mutations in topoisomerase I (topA) suppress the mukB phenotypes (Sawitzke and Austin 2000), and that $\operatorname{mukB}$ mutants are hypersensitive to inhibitors of DNA gyrase (Weitao et al. 1999; Sawitzke and Austin 2000). These results suggest that MukB may participate in higher-order chromosome folding by modulating DNA topology, a mechanism analogous, if not identical, to that proposed for condensin-mediated chromosome condensation in eukaryotic cells (Kimura and Hirano 1997; Kimura et al. 1999).

The identification and functional characterization of bacterial SMC (and MukB) proteins have provided us with an excellent opportunity to compare and contrast chromosome dynamics between the prokaryotic and eukaryotic systems. For example, it has remained elusive for decades how bacterial nucleoids might be organized at a higher-order level. This problem can now be revisited with the new idea that bacterial SMC/MukB proteins may share a common mechanism of action with the eukaryotic condensin complex. It is important to point out that this idea, in turn, raises another question: does the bacterial chromosome cycle have a process corresponding to sister chromatid cohesion? If the bacterial $\mathrm{SMC} / \mathrm{MukB}$ protein is the common ancestor of the eukaryotic cohesin and condensin complexes, it could play a role in cohesion as well as in condensation. In fact, the localization and movement of nascent DNA clusters in
E. coli support the speculation that a cohesion process exists in bacterial cells (Hiraga et al. 2000; Ohsumi et al. 2001). This putative cohesion may be essential for postreplicative repair as suggested in eukaryotic cells (Sjogren and Nasmyth 2001). If SMC/MukB is involved in this process, then it would further emphasize the mechanistic similarity between the bacterial and eukaryotic chromosome cycle. In eukaryotic cells, the four major events of the chromosome cycle (duplication, cohesion, condensation, and separation) are functionally coordinated with each other, but are temporally separated and occur at discrete stages of the cell cycle (Fig. 3B). In the bacterial chromosome cycle, these events take place simultaneously in a cell (Fig. 3A). Despite the outward differences in regulation, the mechanistic parallel between the two systems is obvious. For example, bacterial SMC proteins may facilitate separation and segregation of nucleoids by pulling and compacting them into the cell poles (Sawitzke and Austin 2000). This could accompany the loss of putative cohesion, or act in concert with extrusion of nascent DNAs by the replication machinery (Lemon and Grossman 1998). The analogous process in the eukaryotic chromosome cycle is metaphase chromosome condensation, in which sister chromatids are partially separated (or resolved) by condensin-mediated compaction and the accompanying loss of cohesin. The final separation, which is triggered by the cleavage of cohesin at anaphase, uses eukaryote-specific machinery, the mitotic spindle. This idea would explain the ancient origin of SMC-mediated chromosome separation/segregation and the apparent lack of the spindle apparatus in bacterial cells. Further genetic, biochemical, and cell biological studies will be required to test and extend this idea and to enhance our understanding of the evolutionary origins of chromosomal dynamics.

\section{Molecular mechanisms of SMC actions: toward a unified view}

As discussed above, SMC proteins play highly diverse functions in regulating chromosome dynamics in eukaryotic cells, including chromosome condensation, sister chromatid cohesion, recombinational repair, and global gene repression. What do these seemingly different chromosomal processes have in common? How do SMC proteins support these processes at a mechanistic level? How similar and how different are the actions of bacterial and eukaryotic SMC proteins? In this section, an attempt is made to answer these questions from a mechanistic point of view.

\section{The ATP-binding and hydrolysis cycle of SMC proteins}

An early sequence analysis pointed out that all SMC proteins share a unique motif (called the signature motif or the C motif) that is highly conserved among members of the ATP-binding cassette (ABC) superfamily (Saitoh et al. 1994). A recent crystallographic study has shown that 


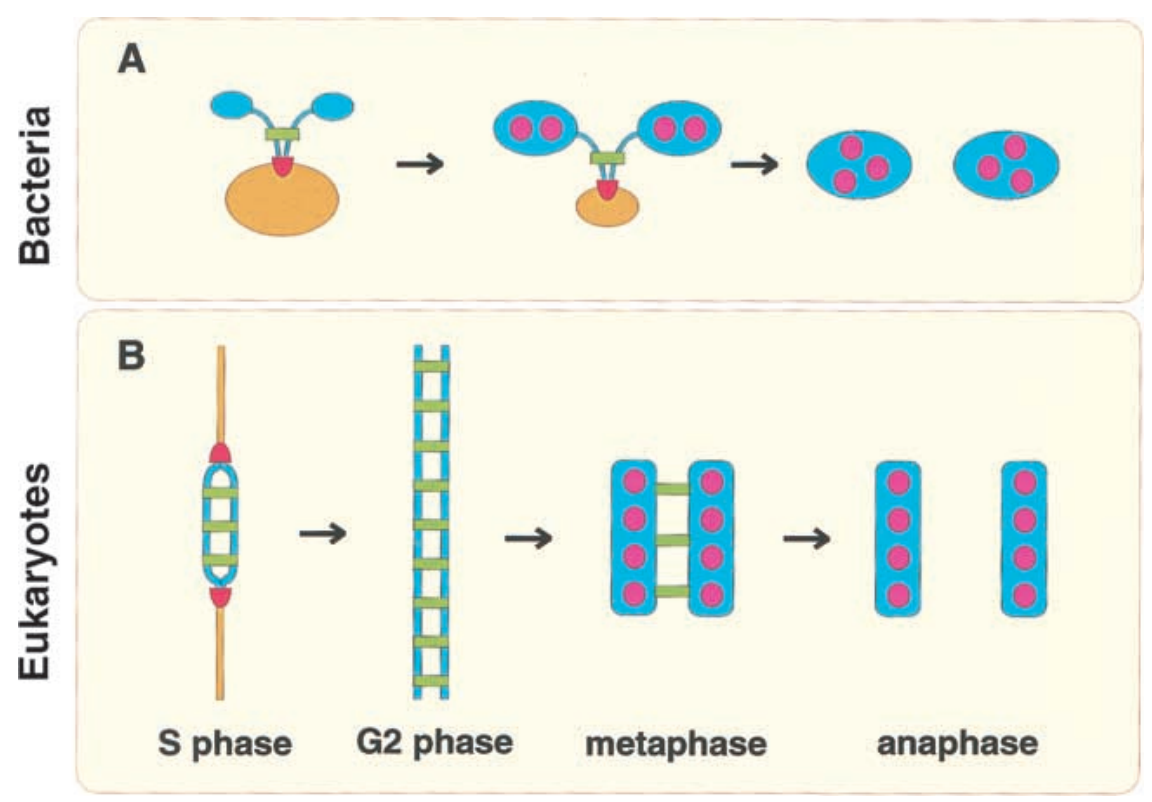

Figure 3. Roles of SMC proteins in the bacterial and eukaryotic chromosome cycle. (A) In bacterial cells, chromosome replication and segregation take place simultaneously. Chromosome separation and segregation are facilitated by a condensin-like function of SMC proteins (magenta). SMC proteins with a putative cohesin-like function (green) may also be involved. Replicated and unreplicated chromosomal regions are shown in blue and orange, respectively. Replication forks are indicated in black. (B) In eukaryotic cells, each chromosomal event occurs at a discrete stage of the cell cycle. When chromosomal DNA is duplicated during S phase, sister chromatid cohesion is established by the action of cohe$\sin$ (green). The linkage between sister chromatids is maintained during $\mathrm{G}_{2}$ phase, and is partially dissolved by metaphase to allow chromosome condensation mediated by condensin (magenta). This partial separation (or resolution) step is most similar to chromosome partitioning in bacterial cells. The full separation of sister chromatids is triggered at the onset of anaphase and is completed by the action of the mitotic spindle (data not shown). the catalytic domain of an SMC protein, composed of the $\mathrm{N}$ and $\mathrm{C}$ termini, indeed displays a protein fold similar to that of the corresponding domains of ABC ATPases (Fig. 4A; Lowe et al. 2001). Therefore, SMC proteins belong to this large superfamily of ATPases, members of which include numerous ABC transporters (for review, see Hol- land and Blight 1999) and the double-strand-break repair protein Rad50 (for review, see Haber 1998). A common structural feature of these ABC ATPases is that each functional complex contains two catalytic domains (also called nucleotide-binding domains, NBDs). In the case of ABC transporters, the two NBDs cooperatively modulate
A

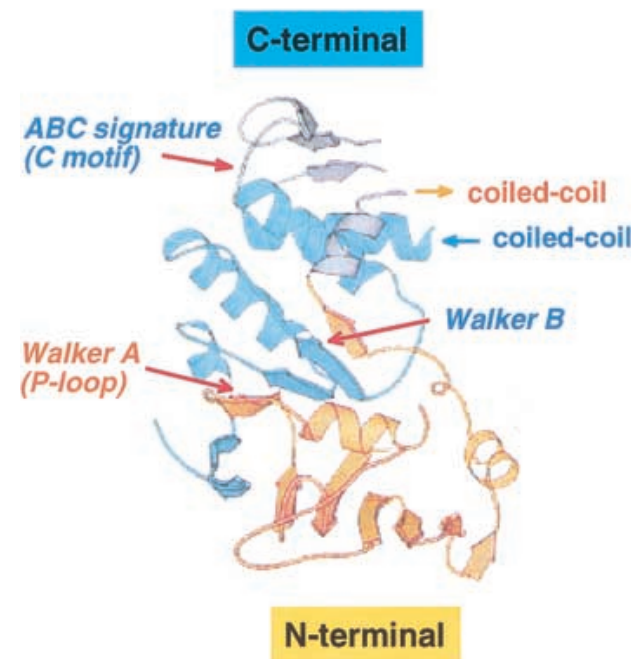

B

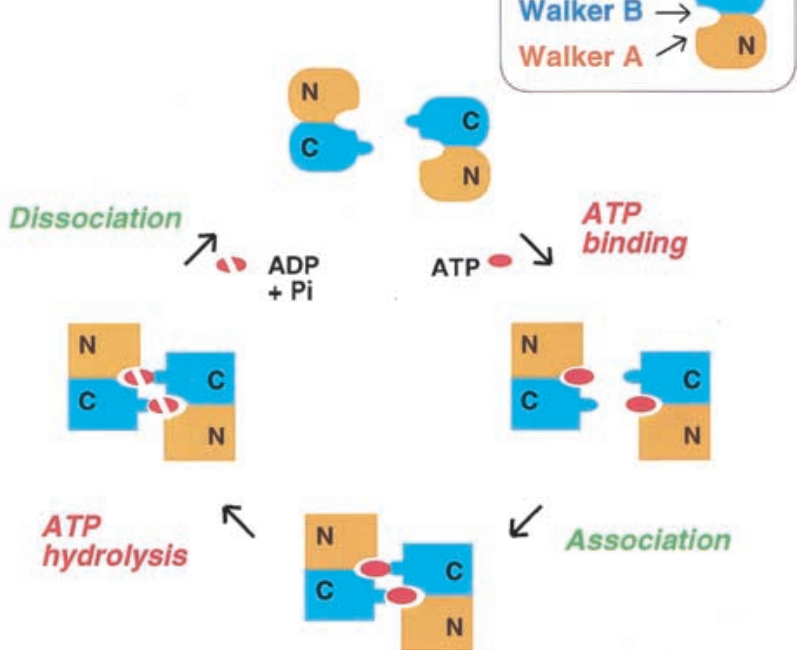

Figure 4. SMC proteins belong to the ABC ATPase superfamily. (A) Crystal structure of an SMC catalytic domain consisting of the $\mathrm{N}$-terminal (orange) and C-terminal (blue) sequences (reproduced from J. Mol. Biol., 2001, 306: 25-35, by copyright permission of Academic Press). Three important motifs, Walker A, Walker B, and ABC signature (or C motif), are indicated. (B) Hypothetical ATP-binding and hydrolysis cycle of SMC proteins. SMC ATPase may act as a composite ATPase, in which hydrolysis of ATP is triggered by the interaction between the two catalytic domains. 
neighboring transmembrane domains (TMDs) so that small molecules (e.g., ions, amino acids, and lipids) are actively transported across the cellular membrane. Rad50 forms a homodimer whose two-armed structure is very similar to that of SMC dimers (Hopfner et al. 2000; Anderson et al. 2001). Biochemical data suggest that the two catalytic domains of ABC proteins functionally interact with each other to modulate their ATPase activity. It is highly controversial, however, how this might be achieved at the structural level, because most of the protein crystals solved to date are monomeric forms. One important exception is Rad50 (Hopfner et al. 2000). The crystal structure of a nucleotide-bound form of Rad50 shows that ATP binding induces the association of the two catalytic domains and thereby creates a DNAbinding surface. Two ATP molecules are sandwiched in the interface of the catalytic domains, and their hydrolysis requires a proper interaction of the two catalytic domains. It remains to be determined to what extent the information deduced from the Rad50 structure may be applicable to the action of SMC ATPases, because the biochemical activities of Rad50 and SMCs are substantially different. For example, unlike Rad50, neither ATP binding nor dimerization of the catalytic domains is essential for the DNA-binding activity of SMC proteins (Hirano and Hirano 1998; Hirano et al. 2001). Despite these seemingly different functional characters, it would be reasonable to speculate that Rad50 and SMCs share a common scheme of ATP binding and hydrolysis (Fig. 4B). This type of composite ATP-binding site is also found in the mismatch DNA repair protein MutS (Junop et al. 2001), and may represent a widespread feature of an even larger group of ATPases beyond the canonical ABC proteins. Most recently, the crystal structure of a dimeric form of the bacterial ABC transporter MsbA has been determined (Chang and Roth 2001). The V-shaped arrangement of the two transmembrane domains is reminiscent of the two-armed structure of SMCs and Rad50, further suggesting a common mechanism of action of the ABC ATPases. (It should be added, however, that the dimer interface deduced from the current MsbA crystal is different from that of $\operatorname{Rad} 50$.

\section{Bimodal activation model of SMC ATPase}

What is the role of the ATP-binding and hydrolysis cycle in the actions of SMC proteins? If the two catalytic domains of SMC proteins constitute a composite ATPase, then the two-armed, symmetrical structure predicts, in principle, two distinct modes of ATPase activation. First, closing of the arms would trigger ATP hydrolysis by allowing an interaction between the two catalytic domains within a dimer (intramolecular mode; Fig. 5A). Second, opening of the arms would allow the catalytic domains of one dimer to interact with those of a neighboring dimer, thereby causing ATP hydrolysis (intermolecular mode; Fig. 5B). A recent mechanistic analysis of the BsSMC homodimer has provided evidence that both activation modes may, indeed, be used by SMC proteins (Hirano et al. 2001). In the absence of DNA, no dimer- dimer interaction is observed and ATP hydrolysis is activated only by the intramolecular mode. When BsSMC binds to DNA, ATP promotes a dimer-dimer interaction, which, in turn, activates their DNA-dependent ATPase by the intermolecular mode. This bimodal activation model provides a natural explanation for the unique, two-armed structure of SMC proteins, although its physiological significance in bacterial cells needs to be explored. The model further emphasizes the functional flexibility and large potential of the unique design of this class of ABC ATPases.

\section{Molecular actions of condensin and cohesin}

The bimodal activation model predicts that opening and closing of the coiled-coil arms make fundamental contributions to the actions of SMC proteins. How can this idea be extended to explain the actions of eukaryotic SMC protein complexes? A recent biochemical study has revealed that purified condensin and cohesin show strikingly different DNA-binding properties in vitro (Losada and Hirano 2001a). In a simple gel-shift assay, for example, condensin produces a discrete set of shifted bands, whereas cohesin induces the formation of large protein-DNA aggregates. These results are consistent with our previous hypothesis that condensin might function as an intramolecular DNA cross-linker that folds a single DNA molecule, whereas cohesin might act as an intermolecular DNA cross-linker that holds two different DNA segments together (Hirano 1999). An important mechanistic question is how condensin and cohesin are able to distinguish between the intramolecular and intermolecular modes of interaction with DNA. One possibility is that different conformations of the SMC subunits confer the two different modes of DNA interactions. For instance, the arms of condensin may primarily be closed, and the action of the two catalytic domains of SMC2-SMC4 would be restricted so that they can only bind to contiguous DNA segments (Fig. 5C). On the other hand, an open conformation of cohesin's arms may allow the two catalytic domains of SMC1-SMC3 to bind to two noncontiguous DNA segments. This could further be facilitated or strengthened by the protein-protein interaction between two cohesin complexes (Fig. 5D). An additional prediction of the bimodal activation model is that the dynamic DNA interactions of condensin and cohesin may be regulated primarily by the intramolecular and intermolecular modes of ATPase cycle, respectively (Fig. 5C,D). We suggest that the two eukaryotic SMC protein complexes are structurally and functionally differentiated from the prototype of SMC proteins (e.g., BsSMC). It is of great interest to test whether the establishment and dissolution of cohesion is functionally coupled with the ATP-binding and hydrolysis cycle of the cohesin complex.

The condensin complex actively reconfigures the DNA structure by using the energy of ATP hydrolysis in vitro. Two different assays have been used to characterize these activities. In the presence of topoisomerase I, condensin introduces positive supercoils into relaxed 


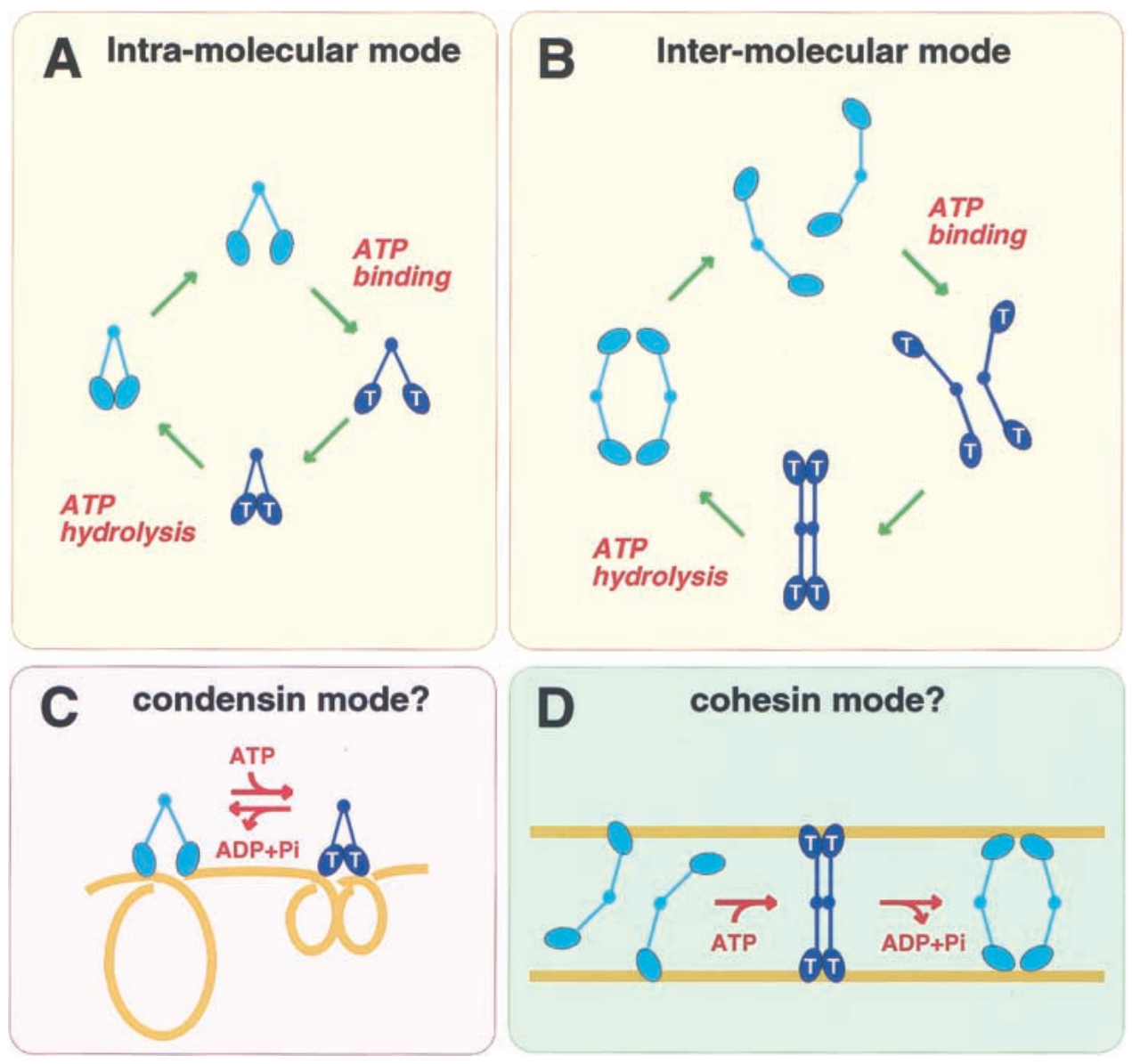

Figure 5. Dynamic actions of SMC ATPases supported by the two-armed structure. $(A, B)$ Bimodal activation of SMC ATPase. Closing of the arms triggers ATP hydrolysis by allowing the interaction between the two catalytic domains within an SMC dimer $(A$, intramolecular mode). Opening of the arms allows the catalytic domains of one dimer to interact with those of another dimer, which, in turn, activates ATP hydrolysis ( $B$, intermolecular mode). $(C, D)$ Hypothetical actions of condensin and cohesin. (C) Condensin may primarily use the intramolecular ATPase mode to compact a single DNA molecule. $(D)$ Cohesin may use the intermolecular ATPase mode to promote and modulate interactions between two different DNA molecules.

circular DNA (Kimura and Hirano 1997; Kimura et al. 2001). In the presence of topoisomerase II, condensin converts nicked circular DNA into positively knotted forms (Kimura et al. 1999, 2001). Neither of these activities can be supported by the core SMC2-SMC4 dimer alone, suggesting that the non-SMC subunits are actively involved in these reactions (Kimura and Hirano 2000). Although these activities are compatible with the action of condensin predicted above, a full understanding of the mechanism requires a combination of structural and biophysical approaches including electron microscopy and single-molecule manipulations. Much less is known about the molecular action of the cohesin complex. In the presence of topoisomerase II, cohesin directs intermolecular catenation of DNA as opposed to intramolecular knotting promoted by condensin (Losada and Hirano 2001a). This action of cohesin, however, does not require ATP, and purified cohesin shows very low, if any, ATPase activity (A. Losada and T. Hirano, unpubl.). One possibility is that an additional factor(s) is required for stimulating cohesin's ATPase and for reconstituting its hypothetical ATP-dependent activities. Candidates for such factors may include Scc2/Mis4 and Pds5/BimD/ Spo76, two HEAT-containing proteins implicated in establishing cohesion in concert with cohesin. Very little is known at present about the biochemical properties of the SMC5-SMC6 complex (Fousteri and Lehmann 2000) or the MukBEF complex (Yamazoe et al. 1999).

\section{Future directions}

The first genetic study of an SMC protein in yeast was published only eight years ago (Strunnikov et al. 1993). Since then, we have witnessed unusually rapid progress in this research field and enjoyed a very rich harvest, which has completely changed our view of chromosome dynamics. There is no doubt that SMC proteins are central to a broad spectrum of higher-order chromosome dynamics in organisms ranging from bacteria to humans. Our present knowledge appears to be only the tip of the iceberg, however, and many important and fundamental questions remain to be answered. First, for historical rea- 
sons, the mitotic function of the SMC protein complexes has been emphasized thus far. Their interphase functions in recombination and gene regulation need to be explored more rigorously and more systematically. Second, our understanding of the meiotic functions of the SMC protein complexes is far from complete. For instance, surprisingly little is known about the potential role of condensin in meiotic chromosome morphogenesis. Third, there remains a huge gap in our understanding of the bacterial and eukaryotic chromosome cycles. Information from the simple model systems will continuously provide vital hints to the more sophisticated actions of eukaryotic SMC protein complexes. Fourth and finally, despite the accumulating information on their cellular functions, we are only beginning to understand the mechanics of this unique class of two-armed ATPases. Although SMC proteins were originally predicted to be chromatin motors, it is now clear that they represent a completely novel type of protein machine. Future work should integrate knowledge from different approaches including genetics, cell biology, biochemistry, structural biology, and biophysics, and thereby help unveil the highly dynamic nature of chromosome structure and function. SMC proteins indeed possess the secret of this fundamental problem because they always lie at the heart of the chromosomes.

\section{Acknowledgments}

We thank members of the Hirano laboratory for critically reading the manuscript. The work from the author's laboratory was supported by grants from the National Institutes of Health, the Pew Scholars Program in the Biomedical Sciences, and the Human Frontier Science Program.

\section{Note added in proof}

A recent study in $S$. pombe reveals a direct interaction between Swi6 and the cohesin subunit Psc3, thereby shedding further lights on the mechanism by which a subpopulation of cohesin is specifically recruited to pericentromeric heterochromatin (Nonaka, N., Kitajima, T., Yokobayashi, S., Xiao, G., Yamamoto, M., Grewal, S.I.S., and Watanabe, Y. 2002. Recruitment of cohesin to heterochromatic regions by Swi6/HP1 in fission yeast. Nat. Cell Biol. 4: 89-93). Another study by electron microscopy shows that condensin and cohesin display remarkably different arm conformations, supporting the idea that the two SMC protein complexes are structurally differentiated to mediate their specialized biochemical and cellular functions (Anderson, D.E., Losada, A., Erickson, H.P., and Hirano, T. 2002. Condensin and cohesin display different arm conformations with characteristic hinge angles. J. Cell Biol., in press).

\section{References}

Adams, R.R., Wheatley, S.P., Gouldsworthy, A.M., KandelsLewis, S.E., Carmena, M., Smythe, C., Gerloff, D.L., and Earnshaw, W.C. 2000. INCENP binds the aurora-related kinase AIRK2 and is required to target it to chromosomes, the central spindle and cleavage furrow. Curr. Biol. 10: 10751078.
Adams, R.R., Eckley, D.M., Vagnarelli, P., Wheatley, S.P., Gerloff, D.L., Mackay, A.M., Svingen, P.A., Kaufmann, S.H., and Earnshaw, W.C. 2001a. Human INCENP colocalizes with the aurora-B/AIRK2 kinase on chromosomes and is overexpressed in tumor cells. Chromosoma 110: 65-74.

Adams, R.R., Maiato, H., Earnshaw, W.C., and Carmena, M. 2001b. Essential roles of Drosophila inner centromere protein (INCENP) and aurora B in histone H3 phosphorylation, metaphase chromosome alignment, kinetochore disjunction, and chromosome segregation. I. Cell Biol. 153: 865880.

Alexandru, G., Uhlmann, F., Mechtler, K., Poupart, M.-A., and Nasmyth, K. 2001. Phosphorylation of the cohesin subunit Scc1 by Polo/Cdc5 kinase regulates sister chromatid cohesion in yeast. Cell 105: 459-472.

Anderson, D.E., Trujillo, K.M., Sung, P., and Erickson, H.P. 2001. Structure of the Rad50/Mre11 DNA repair complex from Saccharomyces cerevisiae by electron microscopy. $I$. Biol. Chem. 276: 37027-37033.

Bai, X., Peirson, B.N., Dong, F., Xue, C., and Makaroff, C.A. 1999. Isolation and characterization of SYN1, a RAD21-like gene essential for meiosis in Arabidopsis. Plant Cell 11: 417-430.

Bernard, P., Maure, J.-F., Partridge, J.F., Genier, S., Javerzat, J.-P., and Allshire, R.C. 2001. Requirement of heterochromatin for cohesion at centromeres. Science 294: 2539-2542.

Bhat, M.A., Philp, A.V., Glover, D.M., and Bellen, H.J. 1996. Chromatid segregation at anaphase requires the barren product, a novel chromosome associated protein that interacts with topoisomerase II. Cell 87: 1103-1114.

Bhatt, A.M., Lister, C., Page, T., Fransz, P., Findlay, K., Jones, G.H., Dickinson, H.G., and Dean, C. 1999. The DIF1 gene of Arabidopsis is required for meiotic chromosome segregation and belongs to the REC8/RAD21 cohesin gene family. Plant I. 19: 463-472.

Blat, Y. and Kleckner, N. 1999. Cohesins bind to preferential sites along yeast chromosome III, with differential regulation along arms versus the centric region. Cell 98: 249-259.

Britton, R.A. and Grossman, A.D. 1999. Synthetic lethal phenotypes caused by mutations affecting chromosome partitioning in Bacillus subtilis. J. Bacteriol. 181: 5860-5864.

Britton, R.A., Lin, D.C.-H., and Grossman, A.D. 1998. Characterization of a prokaryotic SMC protein involved in chromosome partitioning. Genes \& Dev. 12: 1254-1259.

Buonomo, S.B.C., Clyne, R.K., Fuchs, J., Loidl, J., Uhlmann, F., and Nasmyth, K. 2000. Disjunction of homologous chromosomes in meiosis I depends on proteolytic cleavage of the meiotic cohesin Rec8 by separin. Cell 103: 387-398.

Cabello, O.A., Eliseeva, E., He, W., Youssoufian, H., Plon, S.E., Brinkley, B.R., and Belmont, J.W. 2001. Cell cycle-dependent expression and nucleolar localization of hCAP-H. Mol. Biol. Cell 12: 3527-3537.

Chang, G. and Roth, C.B. 2001. Structure of MsbA from E. coli: A homolog of the multidrug resistance ATP binding cassette (ABC) transporters. Science 293: 1793-1800.

Ciosk, R., Shirayama, M., Shevchenko, A., Tanaka, T., Toth, A., Shevchenko, A., and Nasmyth, K. 2000. Cohesin's binding to chromosomes depends on a separate complex consisting of Scc2 and Scc4 proteins. Mol. Cell 5: 243-254.

Cobbe, N. and Heck, M.M. 2000. SMCs in the world of chromosome biology: From prokaryotes to higher eukaryotes. I. Struct. Biol. 129: 123-143.

Collas, P., Le Guellec, K., and Tasken, K. 1999. The A-kinase anchoring protein AKAP95 is a multivalent protein with a key role in chromatin condensation at mitosis. J. Cell Biol. 147: 1167-1179. 
Dej, K.J. and Orr-Weaver, T.L. 2000. Separation anxiety at the centromere. Trends Cell Biol. 10: 392-399.

de la Barre, A.-E., Gerson, V., Gout, S., Creaven, M., Allis, C.D., and Dimitrov, S. 2000. Core histone N-termini play an essential role in mitotic chromosome condensation. EMBO $\mathrm{J}$. 19: $379-391$.

De Souza, C.P.C., Osmani, A.H., Wu, L.-P., Spotts, J.L., and Osmani, S.A. 2000. Mitotic histone H3 phosphorylation by the NIMA kinase in Aspergillus nidulans. Cell 102: 293302.

Eijpe, M., Heyting, C., Gross, B., and Jessberger, R. 2000. Association of mammalian SMC1 and SMC3 proteins with meiotic chromosomes and synaptonemal complexes. J. Cell Sci. 113: 673-682.

Feng, H., Zhong, W., Punkosdy, G., Gu, S., Zhou, L., Seabolt, E.K., and Kipreos, E.T. 1999. CUL-2 is required for the G1to-S-phase transition and mitotic chromosome condensation in Caenorhabditis elegans. Nat. Cell Biol. 1: 486-492.

Fousteri, M. and Lehmann, A.R. 2000. A novel SMC protein complex in Schizosaccharomyces pombe contains the Rad18 DNA repair protein. EMBO J. 19: 1691-1702.

Freeman, L., Aragon-Alcaide, L., and Strunnikov, A.V. 2000. The condensin complex governs chromosome condensation and mitotic transmission of rDNA. I. Cell Biol. 149: 811824.

Giet, R. and Glover, D.M. 2001. Drosophila aurora B kinase is required for histone $\mathrm{H} 3$ phosphorylation and condensin recruitment during chromosome condensation and to organize the central spindle during cytokinesis. J. Cell Biol. 152: 669681.

Goto, H., Tomono, Y., Ajiro, K., Kosako, H., Fujita, M., Sakurai, M., Okawa, K., Iwamatsu, A., Okigaki, T., Takahashi, T., et al. 1999. Identification of a novel phosphorylation site on histone coupled with mitotic chromosome condensation. J. Biol. Chem. 274: 25543-25549.

Graumann, P.L. 2000. Bacillus subtilis SMC is required for proper arrangement of the chromosome and for efficient segregation of replication termini but not for bipolar movement of newly duplicated origin regions. J. Bacteriol. 182: 6463 6471.

Graumann, P.L., Losick, R., and Strunnikov, A.V. 1998. Subcellular localization of Bacillus subtilis SMC, a protein in volved in chromosome condensation and segregation. J. Bacteriol. 180: 5749-5755.

Guacci, V., Koshland, D., and Strunnikov, A. 1997. A direct link between sister chromatid cohesion and chromosome condensation revealed through the analysis of $M C D 1$ in $S$. cerevisiae. Cell 91: 47-57.

Haber, J.E. 1998. The many interfaces of Mre11. Cell 95: 585586.

Hanna, J., Kroll, E.S., Lundblad, V., and Spencer, F.A. 2001. Saccharomyces cerevisiae CTF18 and CTF4 are required for sister chromatid cohesion. Mol. Cell. Biol. 21: 3144-3158.

Hari, K.L., Cook, K.R., and Karpen, G.H. 2001. The Drosophila Su(var)2-10 locus regulates chromosome structure and function and encodes a member of the PIAS protein family. Genes \& Dev. 15: 1334-1348.

Hartman, T., Stead, D., Koshland, D., and Guacci, V. 2000. Pds5 is an essential chromosomal protein required for both sister chromatid cohesion and condensation in Saccharomyces cerevisiae. J. Cell Biol. 151: 613-626.

Hauf, S., Waizenegger, I.C., and Peters, J.-M. 2001. Cohesin cleavage by separase required for anaphase and cytokinesis in human cells. Science 293: 1320-1323.

Hiraga, S. 2000. Dynamic localization of bacterial and plasmid chromosomes. Annu. Rev. Genet. 34: 21-59.
Hiraga, S., Ichinose, C., Onogi, T., Niki, H., and Yamazoe, M 2000. Bidirectional migration of SeqA-bound hemimethylated DNA clusters and pairing of oriC copies in Escherichia coli. Genes Cells 5: 327-341.

Hirano, M. and Hirano, T. 1998. ATP-dependent aggregation of single-stranded DNA by a bacterial SMC homodimer. EMBO J. 17: 7139-7148.

Hirano, M., Anderson, D.E., Erickson, H.P., and Hirano, T. 2001. Bimodal activation of SMC ATPase by intra- and intermolecular interactions. EMBO J. 20: 3238-3250.

Hirano, T. 1999. SMC-mediated chromosome mechanics: A conserved scheme from bacteria to vertebrates? Genes \& Dev. 13: 11-19.

2000. Chromosome cohesion, condensation and separation. Annu. Rev. Biochem. 69: 115-144.

Hirano, T. and Mitchison, T.J. 1993. Topoisomerase II does not play a scaffolding role in the organization of mitotic chromosomes assembled in Xenopus egg extracts. I. Cell Biol. 120: $601-612$.

. 1994. A heterodimeric coiled-coil protein required for mitotic chromosome condensation in vitro. Cell 79: 449-458.

Hirano, T., Kobayashi, R., and Hirano, M. 1997. Condensins, chromosome condensation protein complexes containing XCAP-C, XCAP-E and a Xenopus homolog of the Drosophila Barren protein. Cell 89: 511-521.

Holland, I.B. and Blight, M.A. 1999. ABC-ATPases, adaptable energy generators fuelling transmembrane movement of a variety of molecules in organisms from bacteria to humans. J. Mol. Biol. 293: 381-399.

Holt, C.L. and May, G.S. 1996. An extragenic suppressor of the mitosis-defective bimD6 mutation of Aspergillus nidulans codes for a chromosome scaffold protein. Genetics 142: 777-787.

Hopfner, K.-P., Karcher, A., Shin, D.S., Craig, L., Arthur, L.M., Carney, J.P., and Tainer, J.A. 2000. Structural biology of Rad50 ATPase: ATP-driven conformational control in DNA double-strand break repair and the ABC-ATPase superfamily. Cell 101: 789-800.

Hoque, M.T. and Ishikawa, F. 2001. Human chromatid cohesion component hRad21 is phosphorylated in $\mathrm{M}$ phase and associated with metaphase centromeres. J. Biol. Chem. 276: 5059-5067.

Hsu, J.-Y., Sun, Z.-W., Li, X., Reuben, M., Tatchell, K., Bishop, D.K., Grushcow, J.M., Brame, C.J., Caldwell, J.A., Hunt, D.F., et al. 2000. Mitotic phosphorylation of histone H3 is governed by Ipl1/aurora kinase and glc7/PP1 phosphatase in budding yeast and nematodes. Cell 102: 279-291.

Jensen, R.B. and Shapiro, L. 1999. The Caulobacter crescentus $s m c$ gene is required for cell cycle progression and chromosome segregation. Proc. Natl. Acad. Sci. 96: 10661-10666.

Johnson, E.S. and Gupta, A.A. 2001. An E3-like factor that promotes SUMO conjugation to the yeast septins. Cell 106: $735-744$

Junop, M.S., Obmolova, G., Rausch, K., Hsieh, P., and Yang, W. 2001. Composite active site of an ABC ATPase: MutS uses ATP to verify mismatch recognition and authorize DNA repair. Mol. Cell 7: 1-12.

Kaitna, S., Mendoza, M., Jantsch-Plunger, V., and Glotzer, M. 2000. Incenp and an Aurora-like kinase form a complex essential for chromosome segregation and efficient completion of cytokinesis. Curr. Biol. 10: 1172-1181.

Kimura, K. and Hirano, T. 1997. ATP-dependent positive supercoiling of DNA by $13 \mathrm{~S}$ condensin: A biochemical implication for chromosome condensation. Cell 90: 625-634.

. 2000. Dual roles of the $11 \mathrm{~S}$ regulatory subcomplex in condensin functions. Proc. Natl. Acad. Sci. 97: 1197211977. 
Kimura, K., Hirano, M., Kobayashi, R., and Hirano, T. 1998. Phosphorylation and activation of $13 \mathrm{~S}$ condensin by cdc2 in vitro. Science 282: 487-490.

Kimura, K., Rybenkov, V.V., Crisona, N.J., Hirano, T., and Cozzarelli, N.R. 1999. 13S condensin actively reconfigures DNA by introducing global positive writhe: Implications for chromosome condensation. Cell 98: 239-248.

Kimura, K., Cuvier, O., and Hirano, T. 2001. Chromosome condensation by a human condensin complex in Xenopus egg extracts. J. Biol. Chem. 276: 5417-5420.

Klein, F., Mahr, P., Galova, M., Buonomo, S.B.C., Michaelis, C., Nairz, K., and Nasmyth, K. 1999. A central role for cohesins in sister chromatid cohesion, formation of axial elements, and recombination during yeast meiosis. Cell 98: $91-$ 103.

Kobe, B. and Kajava, A.V. 2000. When protein folding is simplified to protein coiling: The continuum of solenoid protein structures. Trends Biochem. Sci. 25: 509-515.

Koshland, D. and Strunnikov, A. 1996. Mitotic chromosome condensation. Annu. Rev. Cell Dev. Biol. 12: 305-333.

Krawchuk, M.D., DeVeaux, L.C., and Wahls, W.P. 1999. Meiotic chromosome dynamics dependent upon the rec8, rec10, rec11 genes of the fission yeast Schizosaccharomyces pombe. Genetics 153: 57-68.

Laloraya, S., Guacci, V., and Koshland, D. 2000. Chromosomal addresses of the cohesin component Mcdlp. J. Cell Biol. 151: $1047-1056$

Lavoie, B.D., Tuffo, K.M., Oh, S., Koshland, D., and Holm, C. 2000. Mitotic chromosome condensation requires Brnlp, the yeast homolog of Barren. Mol. Biol. Cell 11: 1293-1304.

Lehmann, A.R., Walicka, M., Griffiths, D.J.F., Murray, J.M., Watts, F.Z., McCready, S., and Carr, A.M. 1995. The rad18 gene of Schizosaccharomyces pombe defines a new subgroup of the SMC superfamily involved in DNA repair. Mol. Cell. Biol. 15: 7067-7080.

Lemon, K.P. and Grossman, A.D. 1998. Localization of bacterial DNA polymerase: Evidence for a factory model of replication. Science 282: 1516-1519.

Lieb, J.D., Albrecht, M.R., Chuang, P.-T., and Meyer, B.J. 1998. MIX-1: An essential component of the C. elegans mitotic machinery executes X-chromosome dosage compensation. Cell 92: 265-277.

Losada, A. and Hirano, T. 2001a. Intermolecular DNA interactions stimulated by the cohesin complex in vitro: Implications for sister chromatid cohesion. Curr. Biol. 11: 268272 .

- 2001b. Shaping the metaphase chromosome: Coordination of cohesion and condensation. BioEssays 23: 924-935.

Losada, A., Hirano, M., and Hirano, T. 1998. Identification of Xenopus SMC protein complexes required for sister chromatid cohesion. Genes \& Dev. 12: 1986-1997.

Losada, A., Yokochi, T., Kobayashi, R., and Hirano, T. 2000. Identification and characterization of SA/Scc3p subunits in the Xenopus and human cohesin complexes. J. Cell Biol. 150: 405-416.

Lowe, J., Cordell, S.C., and van den Ent, F. 2001. Crystal structure of the SMC head domain: An ABC ATPase with 900 residues antiparallel coiled coil inserted. J. Mol. Biol. 306: $25-35$.

Lupo, R., Breiling, A., Bianchi, M.E., and Orlando, V. 2001. Drosophila chromosome condensation proteins topoisomerase II and barren colocalize with polycomb and maintain Fab-7 PRE silencing. Mol. Cell 7: 127-136.

MacCallum, D.E., Losada, A., Kobayashi, R., and Hirano, T. 2002. ISWI remodeling complexes in Xenopus egg extracts: Identification as major chromosomal components that are regulated by INCENP-aurora B. Mol. Biol. Cell 13: (in press).

Mayer, M.L., Gygi, S.P., Aebersold, R., and Hieter, P. 2001. Identification of RFC(Ctf18p, Ctf8p, Dcc1p): An alternative RFC complex required for sister chromatid cohesion in $S$. cerevisiae. Mol. Cell 7: 959-970.

Melby, T.E.G., Ciampaglio, C.N., Briscoe, G., and Erickson, H.P. 1998. The symmetrical structure of structural maintenance of chromosomes (SMC) and MukB proteins: Long, antiparallel coiled coils, folded at a flexible hinge. J. Cell Biol. 142: $1595-1604$.

Melchior, F. 2000. SUMO-Nonclassical ubiquitin. Annu. Rev. Cell Dev. Biol. 16: 591-626.

Mengiste, T., Revenkova, E., Bechtold, N., and Paszkowski, J. 1999. An SMC-like protein is required for efficient homologous recombination in Arabidopsis. EMBO J. 18: 45054512.

Meyer, B.J. 2000. Sex in the worm: Counting and compensating X-chromosome dosage. Trends Genet. 16: 247-253.

Michaelis, C., Ciosk, R., and Nasmyth, K. 1997. Cohesins: Chromosomal proteins that prevent premature separation of sister chromatids. Cell 91: 35-45.

Morishita, J., Matusaka, T., Goshima, G., Nakamura, T., Takebe, H., and Yanagida, M. 2001. Birl/cut17 moving from chromosome to spindle upon the loss of cohesion is required for condensation, spindle elongation and repair. Genes Cells 6: 743-763.

Moriya, S., Tsujikawa, E., Hassan, A.K., Asai, K., Kodama, T., and Ogasawara, N. 1998. A Bacillus subtilis gene-encoding protein homologous to eukaryotic SMC motor protein is necessary for chromosome partition and condensation. Mol. Microbiol. 29: 179-187.

Murnion, M.E., Adams, R.A., Callister, D.M., Allis, C.D., Earnshaw, W.C., and Swedlow, J.R. 2001. Chromatin-associated protein phosphatase regulates aurora-B and histone $\mathrm{H} 3$ phosphorylatoin. J. Biol. Chem. 276: 26656-26665.

Nasmyth, K., Peters, J.-M., and Uhlmann, F. 2000. Splitting the chromosome: Cutting the ties that bind sister chromatids. Science 288: 1379-1385.

Neuwald, A.F. and Hirano, T. 2000. HEAT repeats in proteins associated with condensins, cohesins and other chromosome-related complexes. Genome Res. 10: 1445-1452.

Niki, H., Jaffe, A., Imamura, R., Ogura, T., and Hiraga, S. 1991. The new gene mukB codes for a $177 \mathrm{kd}$ protein with coiledcoil domains involved in chromosome partitioning of $E$. coli. EMBO I. 10: 183-193.

Ohsumi, K., Yamazoe, M., and Hiraga, S. 2001. Different localization of SeqA-bound nascent DNA clusters and MukFMukE-MukB complex in Escherichia coli cells. Mol. Microbiol. 40: $835-845$.

Ouspenski, I.I., Cabello, O.A., and Brinkley, B.R. 2000. Chromosome condensation factor Brn $1 \mathrm{p}$ is required for chromatid separation in mitosis. Mol. Biol. Cell 11: 1305-1313.

Panizza, S., Tanaka, T., Hochwagen, A., Eisenhaber, F., and Nasmyth, K. 2000. Pds5 cooperates with cohesin in maintaining sister chromatid cohesion. Curr. Biol. 10: 1557-1564.

Parisi, S., McKay, M.J., Molnar, M., Thompson, M.A., van der Spec, P.J., van Drunen-Schoenmaker, E., Kanaar, R., Lehmann, E., Hoejimakers, J.H.J., and Kohli, J. 1999. Rec8, a meiotic recombination and sister chromatid cohesion phosphoprotein of the Rad21p family conserved from fission yeast to humans. Mol. Cell. Biol. 19: 3515-3528.

Pasierbek, P., Jantsch, M., Melcher, M., Schleiffer, A., Schweizer, D., and Loidl, J. 2001. A Caenorhabditis elegans cohesion protein with functions in meiotic chromosome pairing and disjunction. Genes \& Dev. 15: 1349-1360. 
Pelttari, J., Hoja, M.R., Yuan, L., Liu, J.G., Brundell, E., Moens, P., Santucci-Darmanin, S., Jessberger, R., Barbero, J.L., Heyting, C., et al. 2001. A meiotic chromosomal core consisting of cohesin complex proteins recruits DNA recombination proteins and promotes synapsis in the absence of an axial element in mammalian meiotic cells. Mol. Cell. Biol. 21: 5667-5677.

Pezzi, N., Prieto, I., Kremer, L., Perez Jurado, L.A., Valero, C., Del Mazo, J., Martinez-A, C., and Barbero, J.L. 2000. STAG3, a novel gene encoding a protein involved in meiotic chromosome pairing and location of STAG3-related genes flanking the Williams-Beuren syndrome deletion. FASEB $I$. 14: $581-592$

Prieto, I., Suja, J.A., Pezzi, N., Kremer, L., Martinez-A, C., Rufas, J.S., and Barbero, J.L. 2001. Mammalian STAG3 is a cohesin specific to sister chromatid arms during meiosis I. Nat. Cell Biol. 3: 761-766.

Revenkova, E., Eijpe, M., Heyting, C., Gross, B., and Jessberger, R. 2001. Novel meiosis-specific isoform of mammalian SMC1. Mol. Biol. Cell 21: 6984-6998.

Saitoh, N., Goldberg, I., Wood, E.R., and Earnshaw, W.C. 1994. ScII: An abundant chromosome scaffold protein is a member of a family of putative ATPases with an unusual predicted tertiary structure. J. Cell Biol. 127: 303-318.

Saka, Y., Sutani, T., Yamashita, Y., Saitoh, S., Takeuchi, M., Nakaseko, Y., and Yanagida, M. 1994. Fission yeast cut3 and cut14, members of a ubiquitous protein family, are required for chromosome condensation and segregation in mitosis. EMBO J. 13: 4938-4952.

Sawitzke, J. and Austin, S. 2000. Suppression of chromosome segregation defects of Escherichia coli muk mutants by mutations in topoisomerase I. Proc. Natl. Acad. Sci. 97: 16711676.

Schmiesing, J.A., Gregson, H.C., Zhou, S., and Yokomori, K. 2000. A human condensin complex containing hCAP-ChCAP-E and CNAP1, a homolog of Xenopus XCAP-D2, colocalizes with phosphorylated histone $\mathrm{H} 3$ during the early state of mitotic chromosome condensation. Mol. Cell. Biol. 20: 6996-7006.

Siøgren, C. and Nasmyth, K. 2001. Sister chromatid cohesion is required for postreplicative double-strand break repair in Saccharomyces cerevisiae. Curr. Biol. 11: 991-995.

Skibbens, R.V., Corson, L.B., Koshland, D., and Hieter, P. 1999. Ctf7p is essential for sister chromatid cohesion and links mitotic chromosome structure to the DNA replication machinery. Genes \& Dev. 13: 307-319.

Speliotes, E.K., Uren, A., Vaux, D., and Horvitz, H.R. 2000. The survivin-like C. elegans BIR-1 protein acts with the auroralike kinase AIR-2 to control chromosome behavior and spindle midzone organization. Mol. Cell 6: 211-223.

Stahl, B.D. and Allis, C.D. 2000. The language of covalent histone modifications. Nature 403: 41-45.

Steen, R.L., Cubizolles, F., Le Guellec, K., and Collas, P. 2000. A kinase-anchoring protein (AKAP) 95 recruits human chromosome-associated protein (hCAP)-D2/Eg7 for chromosome condensation in mitotic extract. J. Cell. Biol. 149: 531-536.

Steffensen, S., Coelho, P.A., Cobbe, N., Vass, S., Costa, M., Hassan, B., Prokopenko, S.N., Bellen, H., Heck, M.M.S., and Sunkel, C.E. 2001. A role for Drosophila SMC4 in the resolution of sister chromatids in mitosis. Curr. Biol. 11: 295307.

Strunnikov, A.V., Larionov, V.L., and Koshland, D. 1993. SMC1: An essential yeast gene encoding a putative head-rod-tail protein is required for nuclear division and defines a new ubiquitous family. J. Cell Biol. 123: 1635-1648.

Strunnikov, A.V., Hogan, E., and Koshland, D. 1995. SMC2, a
Saccharomyces cerevisiae gene essential for chromosome segregation and condensation, defines a subgroup within the SMC family. Genes \& Dev. 9: 587-599.

Strunnikov, A.V., Aravind, L., and Koonin, E.V. 2001. Saccharomyces cerevisiae SMT4 encodes an evolutionarily conserved protease with a role in chromosome condensation regulation. Genetics 158: 95-107.

Sumara, I., Vorlaufer, E., Gieffers, C., Peters, B.H., and Peters, J.-M. 2000. Characterization of vertebrate cohesin complexes and their regulation in prophase. I. Cell Biol. 151: 749-761.

Sutani, T., Yuasa, T., Tomonaga, T., Dohmae, N., Takio, K., and Yanagida, M. 1999. Fission yeast condensin complex: Essential roles of non-SMC subunits for condensation and Cdc2 phosphorylation of Cut3/SMC4. Genes \& Dev. 13: 2271-2283.

Tanaka, K., Yonekawa, T., Kawasaki, Y., Kai, M., Furuya, K., Iwasaki, M., Murakami, H., Yanagida, M., and Okayama, H. 2000. Fission yeast Esolp is required for establishing sister chromatid cohesion during S phase. Mol. Cell. Biol. 20: 3459-3469.

Tanaka, K., Hao, Z., Kai, M., and Okayama, H. 2001. Establishment and maintenance of sister chromaid cohesion in fission yeast by a unique mechanism. EMBO J. 20: 5779-5790.

Tanaka, T., Cosma, M.P., Wirth, K., and Nasmyth, K. 1999. Identification of cohesin association sites at centromeres and along chromosome arms. Cell 98: 847-858.

Taylor, E.M., Moghraby, J.S., Lees, J.H., Smit, B., Moens, P.B., and Lehmann, A.R. 2001. Characterization of a novel human SMC heterodimer homologous to the Schizosaccharomyces pombe Rad18/Spr18 complex. Mol. Biol. Cell 12: 1583-1594.

Tomonaga, T., Nagao, K., Kawasaki, Y., Furuya, K., Murakami, A., Morishita, J., Yuasa, T., Sutani, T., Kearsey, S.E., Uhlmann, F., et al. 2000. Characterization of fission yeast cohesin: Essential anaphase proteolysis of Rad21 phosphorylated in the S phase. Genes \& Dev. 14: 2757-2770.

Toth, A., Ciosk, R., Uhlmann, F., Galova, M., Schleiffer, A., and Nasmyth, K. 1999. Yeast cohesion complex requires a conserved protein, Ecolp(Ctf7), to establish cohesion between sister chromatids during DNA replication. Genes \& Dev. 13: 320-333.

Uhlmann, F. and Nasmyth, K. 1998. Cohesion between sister chromatids must be established during DNA replication. Curr. Biol. 8: 1095-1101.

Uhlmann, F., Lottspeich, F., and Nasmyth, K. 1999. Sister-chromatid separation at anaphase onset is promoted by cleavage of the cohesin subunit Scc1. Nature 400: 37-42.

Uhlmann, F., Wernic, D., Poupart, M.-A., Koonin, E.V., and Nasmyth, K. 2000. Cleavage of cohesin by the CD clan protease separin triggers anaphase in yeast. Cell 103: 375-386.

Uren, A.G., Wong, L., Pakusch, M., Fowler, K.J., Burrows, F.J., Vaux, D.L., and Choo, K.H.A. 2000. Survivin and the inner centromere protein INCENP show similar cell-cycle localization and gene knockout phenotype. Curr. Biol. 10: 13191328.

van Heemst, D., James, F., Poggeler, S., Berteaux-Lecellier, V., and Zickler, D. 1999. Spo76p is a conserved chromosome morphogenesis protein that links the mitotic and meiotic programs. Cell 98: 261-271.

van Heemst, D., Kafer, E., John, T., Heyting, C., van Aalderen, M., and Zickler, D. 2001. BimD/Spo76 is at the interface of cell cycle progression, chromosome morphogenesis, and recombination. Proc. Nat1. Acad. Sci. 98: 6267-6272.

Verkade, H.M., Bugg, S.J., Lindsay, H.D., Carr, A.M., and O'Connell, M.J. 1999. Rad18 is required for DNA repair and checkpoint responses in fission yeast. Mol. Biol. Cell 
10: $2905-2918$.

Waizenegger, I.C., Hauf, S., Meinke, A., and Peters, J.-M. 2000. Two distinct pathways remove mammalian cohesin from chromosome arms in prophase and from centromeres in anaphase. Cell 103: 399-410.

Wang, Z., Castaño, I.B., De Las Penas, A., Adams, C., and Christman, M.F. 2000. Pol к, a DNA polymerase required for sister chromatid cohesion. Science 289: 774-779.

Warren, W.D., Steffensen, S., Lin, E., Coelho, P., Loupart, M.-L., Cobbe, N., Lee, J.Y., McKay, M.J., Orr-Weaver, T.L., Heck, M.M.S., et al. 2000. The Drosophila RAD21 cohesin persists at the centromere region in mitosis. Curr. Biol. 10: 14631466.

Watanabe, Y. and Nurse, P. 1999. Cohesin Rec8 is required for reductional chromosome segregation in meiosis. Nature 400: 461-464.

Watanabe, Y., Yokobayashi, S., Yamamoto, M., and Nurse, P. 2001. Premeiotic S phase is linked to reductional chromosome segregation and recombination. Nature 409: 359-363.

Wei, Y., Yu, L., Bowen, J., Gorovsky, M.A., and Allis, C.D. 1999. Phosphorylation of histone H3 is required for proper chromosome condensation and segregation. Cell 97: 99-109.

Weitao, T., Nordstrøm, K., and Dasgupta, S. 1999. Mutual suppression of mukB and seqA phenotypes might arise from their opposing influences on the Escherichia coli nucleoid structure. Mol. Microbiol. 34: 157-168.

Wheatley, S.P., Carvalho, A., Vagnarelli, P., and Earnshaw, W.C. 2001. INCENP is required for proper targeting of Survivin to the centromeres and the anaphase spindle during mitosis. Curr. Biol. 11: 886-890.

Yamazoe, M., Onogi, T., Sunako, Y., Niki, H., Yamanaka, K., Ichimura, T., and Hiraga, S. 1999. Complex formation of MukB, MukE and MukF proteins involved in chromosome partitioning in Escherichia coli. EMBO J. 18: 5873-5884. 


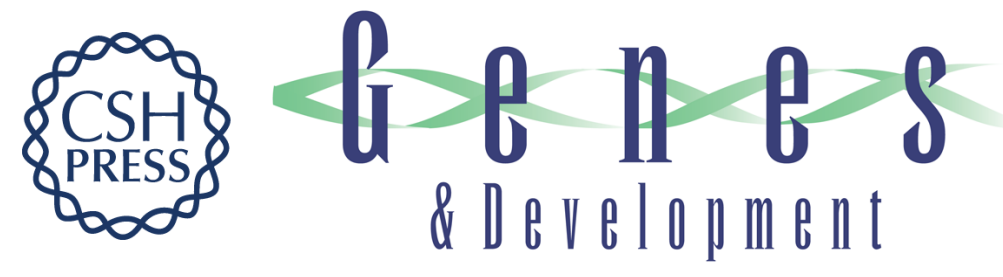

\section{The ABCs of SMC proteins: two-armed ATPases for chromosome condensation, cohesion, and repair}

Tatsuya Hirano

Genes Dev. 2002, 16:

Access the most recent version at doi:10.1101/gad.955102

References This article cites 129 articles, 66 of which can be accessed free at: http://genesdev.cshlp.org/content/16/4/399.full.html\#ref-list-1

License

Email Alerting

Receive free email alerts when new articles cite this article - sign up in the box at the top Service right corner of the article or click here.

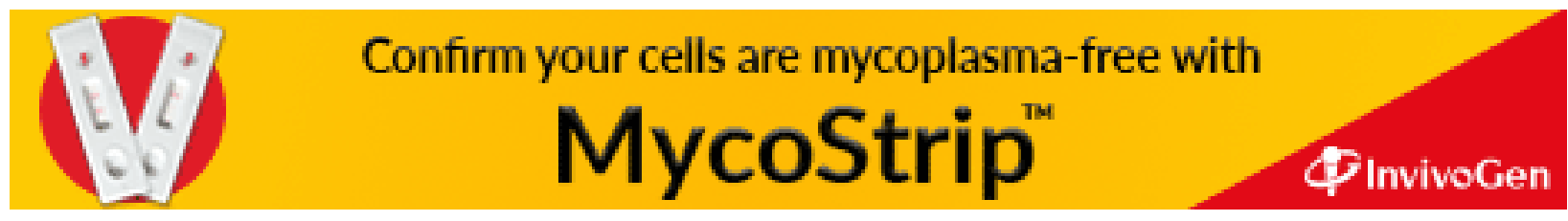

